\title{
Signal Transduction by HDL: Agonists, Receptors, and Signaling Cascades
}

\author{
Jerzy-Roch Nofer
}

\section{Contents}

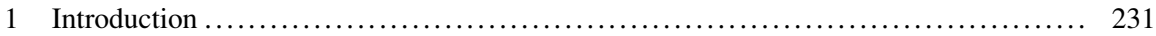

2 ApoA-I-Induced Cell Signaling Directly Mediated by ABCA1 .................. 232

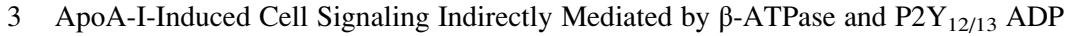

Receptor ...........................................................

4 ApoA-I- and HDL-Induced Cell Signaling Indirectly Mediated by ABCA1 and/or ABCG1 ............................................................. 238

5 HDL-Induced Cell Signaling Mediated by SR-BI ............................. 239

6 HDL-Induced Cell Signaling Mediated by S1P ............................. 243

7 HDL-Induced Cell Signaling: Future Challenges and Opportunities .................. 247

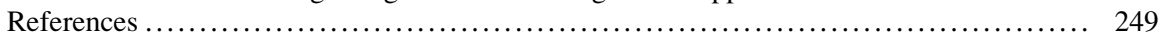

\begin{abstract}
Numerous epidemiologic studies revealed that high-density lipoprotein (HDL) is an important risk factor for coronary heart disease. There are several welldocumented HDL functions such as reversed cholesterol transport, inhibition of inflammation, or inhibition of platelet activation that may account for the atheroprotective effects of this lipoprotein. Mechanistically, these functions are carried out by a direct interaction of HDL particle or its components with receptors localized on the cell surface followed by generation of intracellular signals. Several HDL-associated receptor ligands such as apolipoprotein A-I (apoA-I) or sphingosine-1-phosphate (S1P) have been identified in addition to HDL holoparticles, which interact with surface receptors such as ATP-binding cassette transporter $\mathrm{A} 1$ (ABCA1); $\mathrm{S} 1 \mathrm{P}$ receptor types 1,2 , and $3\left(\mathrm{~S}_{1} \mathrm{P}_{1}, \mathrm{~S}_{1} \mathrm{P}_{2}\right.$, and $\mathrm{S}_{1} \mathrm{P}_{3}$ ); or scavenger receptor type $\mathrm{I}(\mathrm{SR}-\mathrm{BI})$ and activate intracellular
\end{abstract}

\section{J.-R. Nofer ( $\bowtie)$}

Center for Laboratory Medicine, University Hospital Münster, Albert-Schweizer-Campus 1, Geb. A1, D-48149 Münster, Germany

e-mail: nofer@uni-muenster.de 
signaling cascades encompassing kinases, phospholipases, trimeric and small G-proteins, and cytoskeletal proteins such as actin or junctional protein such as connexin43. In addition, depletion of plasma cell cholesterol mediated by ABCA1, ATP-binding cassette transporter G1 (ABCG1), or SR-BI was demonstrated to indirectly inhibit signaling over proinflammatory or proliferation-stimulating receptors such as Toll-like or growth factor receptors. The present review summarizes the current knowledge regarding the HDL-induced signal transduction and its relevance to athero- and cardioprotective effects as well as other physiological effects exerted by HDL.

\section{Keywords}

High-density lipoprotein (HDL) • Apolipoprotein A-I (apoA-I) • ATP-binding cassette transporter A1 (ABCA1) - Scavenger receptor type I (SR-BI) Sphingosine-1-phosphate $(\mathrm{S} 1 \mathrm{P}) \cdot$ Signal transduction

\section{Abbreviations}

ABCA1 ATP-binding cassette transporter A1

ABCG1 ATP-binding cassette transporter G1

AMPK AMP-activated protein kinase

Apo Apolipoprotein

CAMK Calcium/calmodulin-dependent protein kinase

cAMP Cyclic adenosine monophosphate

Cdc42 Cell division control protein 42

CHD Coronary heart disease

CKD Chronic kidney disease

COX-2 Cyclooxygenase-2

DAG diacylglycerol

DHEA Dehydroepiandrosterone

EL Endothelial lipase

eNOS Endothelial nitric oxide synthase

GLUT4 Glucose transporter type 4

GM-CSF Granulocyte macrophage colony-stimulating factor

HDL High-density lipoprotein

HSPC Hematopoietic stem progenitor cells

IL Interleukin

JAK2 Janus kinase 2

JNK c-Jun N-terminal kinase

LCAT Lecithin:cholesterol acyltransferase

LDL Low-density lipoprotein 


$\begin{array}{ll}\text { LPS } & \text { Lipopolysaccharide } \\ \text { MAPK } & \text { Mitogen-activated protein kinase } \\ \text { MCP1 } & \text { Monocyte chemotactic protein 1 } \\ \text { MDCK } & \text { Madin-Darby canine kidney } \\ \text { PC } & \text { Phosphatidylcholine } \\ \text { PC-PLC } & \text { PC-specific phospholipase C } \\ \text { PC-PLD } & \text { PC-specific phospholipase D } \\ \text { PDZK1 } & \text { PDZ domain-containing adaptor protein } \\ \text { PGI } 2 & \text { Prostacyclin } \\ \text { PHD } & \text { Prolyl hydroxylase } \\ \text { PI3K } & \text { Phosphatidylinositol 3-kinase } \\ \text { PI-PLC } & \text { Phosphatidylinositol-specific phospholipase C } \\ \text { PKA } & \text { Protein kinase A } \\ \text { PKC } & \text { Protein kinase C } \\ \text { S1P } & \text { Sphingosine-1-phosphate } \\ \text { SAA } & \text { Serum amyloid A } \\ \text { SDMA } & \text { Symmetric dimethylarginine } \\ \text { SK } & \text { Sphingosine kinase } \\ \text { SPC } & \text { Sphingosylphosphorylcholine } \\ \text { SPM } & \text { Sphingomyelin } \\ \text { SR-BI } & \text { Scavenger receptor type I } \\ \text { STAT3 } & \text { Signal transducer and activator of transcription 3 } \\ \text { TGF } \beta & \text { Transforming growth factor- } \beta \\ \text { TLR } & \text { Toll-like receptor } \\ \text { TNF } \alpha & \text { Tumor necrosis factor } \alpha \\ \beta-A T P a s e & \text { F1F0 ATP synthase } \beta \text {-subunit } \\ & \end{array}$

\section{Introduction}

Innumerable epidemiological studies document the inverse relationship between plasma levels of high-density lipoprotein (HDL) cholesterol and cardiovascular risk. The first hypothesis aiming to mechanistically explain this relationship has been formulated by John Glomset (1968), who proposed that HDL particles take up excess cholesterol in peripheral tissues including atherosclerotically changed arterial walls and transport it to the liver for excretion with bile. The first step in this process termed reverse cholesterol transport encompassed direct interaction between HDL and cell membrane with the subsequent efflux of cholesterol to lipoprotein particles. Early concepts envisioned cholesterol efflux as a purely physicochemical phenomenon with the unidirectional flux of free cholesterol perpetuated by a steady concentration gradient maintained by the activity of lecithin:cholesterol acyltransferase (LCAT) in HDL particles. John F. Oram was the first to note that HDL binding to specific binding sites precedes cholesterol 
efflux and is required for the effective mobilization of free cholesterol from internal pools and its transport to the plasma membrane, where it is available for further transfer to lipoprotein particles (Graham and Oram 1987; Slotte et al. 1987). The seminal discovery of Oram put HDL function in a fully new perspective: ever since then HDL has been perceived not only as a cholesterol transporter but also as hormone-like particle exerting its activities through binding to specific receptors.

Signal transduction occurs when an extracellular signaling molecule activates a surface receptor, which in turn alters intracellular molecules creating a response, which ultimately modifies cellular functions. The recognition of specific HDL binding sites raised immediately a question, whether HDL-cell interaction is linked to signaling events relevant to intracellular cholesterol transport and/or cholesterol efflux. It was Oram again who demonstrated that the exposure of fibroblasts to HDL causes activation of protein kinase $\mathrm{C}$ (PKC), which in turn contributes to translocation of free cholesterol between intracellular membranes and plasmalemma (Mendez et al. 1991). This finding opened a new field of research focusing on dissection of intracellular signaling pathways that are induced by HDL or its constituents and mediate cellular responses to lipoprotein particles. In the following years, several ligands present in HDL and their receptors expressed on the cell surface could be identified that mediate one or more physiological effects attributed to this lipoprotein. In addition, the interaction of HDL with the cell membrane was found to potently modify signal transduction induced by other ligands such as pathogen-associated molecular patterns (PAMPs) or growth factors. This review attempts to summarize the current state of knowledge on HDL-induced signal transduction and its physiological relevance.

\section{$2 \quad$ ApoA-I-Induced Cell Signaling Directly Mediated by ABCA1}

ATP-binding cassette transporter A1 (ABCA1) has been originally identified as a protein defective in familial HDL deficiency (Tangier disease), which is characterized on the molecular level by the failure of apoA-I to mobilize cholesterol from intracellular stores, to induce cholesterol and phospholipid efflux from cells, and ultimately to initiate the maturation of HDL particles and the HDL-mediated reversed cholesterol transport (Bodzioch et al. 1999; Brooks-Wilson et al. 1999; Rust et al. 1999). Early models explaining ABCA1-mediated lipid efflux from cells assumed that this transporter facilitates translocation of cholesterol and phospholipids to the exofacial leaflet of the cell membrane, where they became accessible for the passive transfer to apoA-I. Further studies, however, provided several pieces of evidence suggesting that apoA-I may directly interact with ABCA1 for the effective unloading of cell cholesterol. For instance, cells obtained from patients with Tangier disease displayed reduced number of apoA-I binding sites, whereas increased apoA-I binding to the cell surface could be observed in cells overexpressing full-length cDNA of ABCA1 (Francis et al. 1995; Wang et al. 2000). Moreover, studies utilizing chemical cross-linking indicated that 
apoA-I and ABCA1 are in a very close proximity $(<7 \AA)$ characteristic of ligandreceptor interaction, while naturally occurring mutations in extracellular ABCA1 loops profoundly impaired the cross-linking efficiency (Wang et al. 2000; Fitzgerald et al. 2002, 2004; Chroni et al. 2004). The binding of apoA-I to $\mathrm{ABCA} 1$ was subsequently found to increase the cell content of ABCA1 protein but not the ABCA1 mRNA abundance suggesting that apoA-I-ABCA1 interaction might be critical to ABCA1 stabilization (Wang et al. 2003). Actually, apoA-I was found to promote both the dephosphorylation of the proline-, glutamic acid-, serine-, and threonine-rich (PEST) sequence in the cytoplasmatic domain of ABCA1 and the recruitment of calmodulin to the specific 1-5-8-14 binding motif localized in a close vicinity to the PEST sequence (Wang et al. 2003; Martinez et al. 2003a; Iwamoto et al. 2010). In this manner, apoA-I binding appears to protect ABCA1 against calpain-mediated proteolysis. In addition, lipid-free apoA-I was found to retard ABCA1 degradation mediated by thiol proteases and thereby to facilitate ABCA1 accumulation on the cell surface (Arakawa and Yokoyama 2002).

The recognition of the apoA-I-ABCA1 complex formation and its stabilization as a mechanism protecting against protease-mediated degradation gave rise to the hypothesis that the interaction of apoA-I with ABCA1 may trigger intracellular signaling cascades that modulate ABCA1 levels and/or ABCA1-mediated lipid transport activity in a posttranslational manner (see Fig. 1 for schematic presentation of ABCA1-induced signaling). Early studies documented that apoA-I enhances phosphatidylcholine (PC) turnover in a specific process mediated by a G-proteindependent activation of PC-specific phospholipases C and D (PC-PLC and PC-PLD) (Walter et al. 1995, 1996). Later investigations attributed the apoA-Imediated activation of PC-PLC rather to ABCA1-dependent efflux of sphingomyelin (SPM) and the unspecific depletion of plasma membrane SPM content (Yamauchi et al. 2003). In both cases, stimulation of PC-PLC liberates diacylglycerol (DAG), which is the primary physiological activator of PKC. The activation of both PC-specific phospholipases and PKC seems to be critical for ABCA1-mediated lipid transport. Actually, pharmacological interference with PC-PLC and PC-PLD activation reduced apoA-I-induced cholesterol efflux, whereas exogenous application of their products DAG or phosphatidic acid (PA) partially corrected the cholesterol efflux defect in cells obtained from patients with Tangier disease (Walter et al. 1996; Haidar et al. 2001). Similarly, PKC inhibitors and activators were found, respectively, to block or to enhance apoA-Iinduced cholesterol efflux from various cell types including macrophages, smooth muscle cells, and fibroblasts (Mendez et al. 1991; Li and Yokoyama 1995; Li et al. 1997). More recently, it has been suggested that PKC specifically controls the translocation of cholesterol from acylCoA:cholesterol acyltransferase (ACAT)accessible pools to the as yet poorly defined intracellular cholesterol compartment, where it is available for the ABCA1-mediated release (Yamauchi et al. 2004). In addition, $\mathrm{PKC}$ isoform alpha ( $\mathrm{PKC} \alpha$ ) was found to increase $\mathrm{ABCA} 1$ phosphorylation at as yet unknown serine sites and thereby to enhance it protection against calpain-mediated proteolysis (Yamauchi et al. 2003). Apelin, a bioactive peptide modulating vascular tone and blood pressure and displaying strong antiatherogenic 


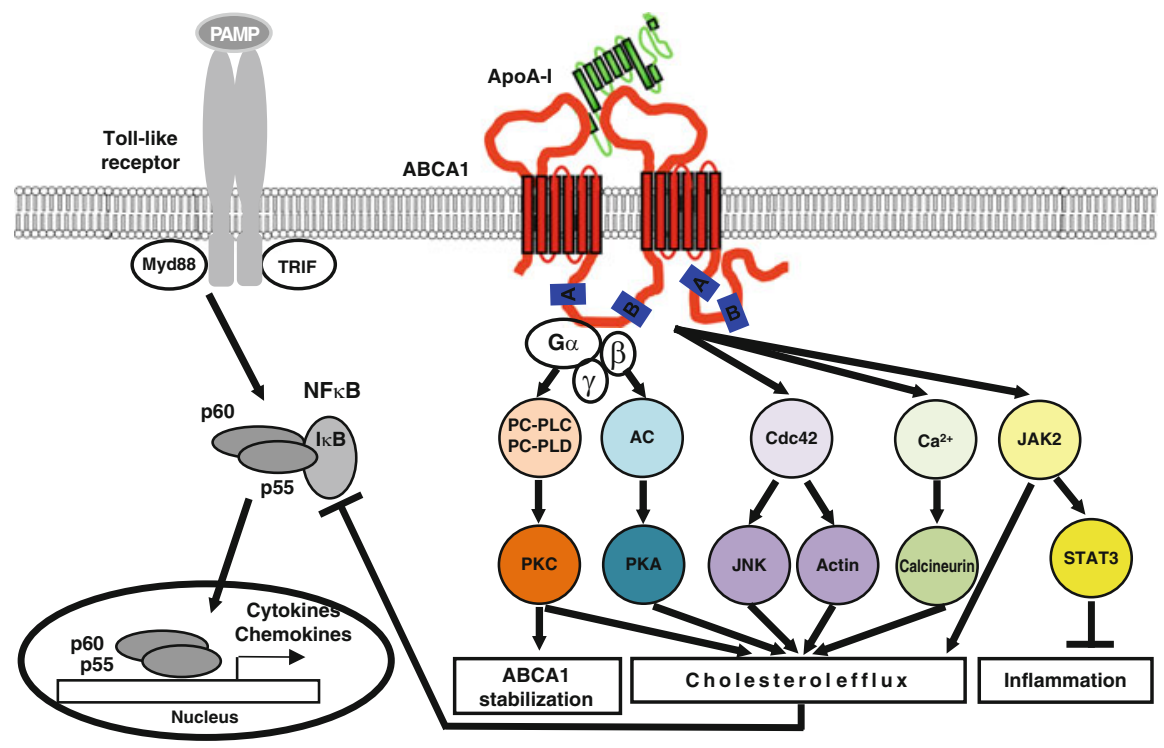

Fig. 1 ApoA-I-induced cell signaling mediated by ABCA1. The binding of apolipoprotein A-I (apoA-I) to lipid transporter ABCA1 induces the trimeric G-protein-mediated activation of phosphatidylcholine-specific phospholipases C and/or D (PC-PLC, PC-PLD) and adenylate cyclase (AC) and the ensuing activation of protein kinases $\mathrm{C}$ and $\mathrm{A}$ (PKC, PKA). Direct interaction of the small G-protein Cdc42 with ABCA1 induces activation of protein kinase JNK and actin polymerization. Direct interaction of Janus kinase-2 (JAK2) with ABCA1 leads to its autophosphorylation and activation as well as activation of transcription factor signal transducer and activator of transcription 3 (STAT3). These effects account for cholesterol efflux-inducing, anti-inflammatory, and ABCA1-stabilizing properties of apoA-I. In addition, ABCA1-mediated cholesterol efflux to apoA-I exerts indirect negative effect on signal transduction mediated by proinflammatory Toll-like receptors and the resulting activation of the transcription factor NF- $\mathrm{\kappa B}$ and expression of cyto- and chemokines. PAMP Pathogen-associated molecular patterns

properties, has been recently found to phosphorylate serine residues in ABCA1 through the PKC $\alpha$ pathway and to inhibit calpain-mediated proteolysis, thereby promoting cholesterol efflux and reducing foam cell formation (Liu et al. 2013a).

ABCA1 is well known to be regulated by cyclic adenosine monophosphate (cAMP) at the transcriptional level, and cAMP responsive elements have been localized in the ABCA1 promoter. The interaction of apoA-I with ABCA1, in turn, was demonstrated to activate adenylate cyclase and to increase cAMP content, while severely reduced apoA-I-induced cAMP production was found in cells obtained from carriers of ABCA1 mutations (Haidar et al. 2004). These observations strongly suggested that cAMP and its downstream target protein kinase A (PKA) may represent an important component of apoA-I/ABCA1-induced signaling cascade regulating intracellular lipid translocation and/or their removal from cells. Actually, PKA phosphorylation sites were identified in the nucleotide binding domains of ABCA1, and the targeted mutation of one of these sites, S2054, significantly impaired apoA-I-mediated lipid efflux (See et al. 2002). Moreover, 
pharmacological modulation of cAMP/PKA signaling pathway exerted profound effects on apoA-I-induced cholesterol efflux. For instance, forskolin, an adenyl cyclase activator, and 8-Br-cAMP, a stable cAMP analog, substantially increased both ABCA1 phosphorylation and cholesterol efflux to apoA-I in wild-type cells and partly corrected the impaired efflux in cells obtained from patients with Tangier disease (Haidar et al. 2002). Similar effects were more recently observed in macrophages treated with Ht31, a PKA-anchoring inhibitor, which robustly elevated PKA activity in the cytoplasm, elevated ABCA1-dependent cholesterol export from cells, and completely reversed foam cell formation (Ma et al. 2011). Conversely, interference with cAMP/PKA signaling accomplished by treating cells with adenyl cyclase inhibitors, PKA inhibitors, or eicosapentaenoic acid, which decreased both cAMP levels and PKA activity, led to concomitant reduction of ABCA1 phosphorylation and ABCA1-mediated cholesterol efflux (Haidar et al. 2002, 2004; $\mathrm{Hu}$ et al. 2009). The molecular mechanisms underlying the stimulatory effect of PKA-mediated ABCA1 phosphorylation on the function of this transporter remain unclear. In contrast to PKC, PKA neither improves ABCA1 stability nor increases apoA-I binding. It seems more likely that phosphorylation of ABCA1 by the CAMP/PKA pathway allows the transporter to assume the conformation favoring more effective lipid translocation across the cell membrane (See et al. 2002).

Janus kinase 2 (JAK2) represents the third kinase, the activity of which is induced by ApoA-I/ABCA1 interaction. Even short exposure of cells expressing ABCA1 to apoA-I produces substantial autophosphorylation of JAK2, which is a prerequisite for further signal transmission to downstream located targets (Tang et al. 2004). Active JAK2 does not seem to directly phosphorylate ABCA1, and it is more likely that it targets an as yet unknown ABCA1 partner protein. Nevertheless, several experimental approaches clearly documented that JAK2 signaling is required for ABCA1 to effectively mediate lipid export from cells. For instance, JAK2-specific inhibitors substantially reduced apoA-I-induced cholesterol efflux, and mutant cells lacking JAK2 exhibited a severely impaired lipid transport (Tang et al. 2004, 2006). In addition, studies exploiting various ABCA1 variants revealed close interdependency between lipid export and JAK2 activation (Vaughan et al. 2009). In contrast to PKC and PKA, JAK2 activation by apoA-I neither promotes ABCA1 stability nor enhances intrinsic lipid translocase activity of the transporter. However, JAK2 activation seems to be critical for the optimum formation of apoA-I/ABCA1 complex, as JAK2-deficient cells exhibit severely impaired apoA-I binding despite normal ABCA1 protein levels (Tang et al. 2006).

JAK2 autophosphorylation is commonly followed by phosphorylation of several downstream targets, out of which signal transducer and activator of transcription 3 (STAT3) represents its canonical target. As ABCA1 possesses STAT3 docking sites, this molecule was an obvious candidate for linking JAK2 with ABCA1 activation. As a matter of fact, apoA-I induces STAT3 phosphorylation (which is tantamount to its activation) in an ABCA1-dependent fashion, and this effect is attenuated in cells expressing ABCA1, in which STAT3 docking sites have been mutated (Tang et al. 2009). However, neither complete STAT3 deficiency nor 
interruption of ABCA1-STAT3 interaction altered ABCA1 capacity to bind apoA-I and to efflux cholesterol from cells (Tang et al. 2009). Hence, apoA-I-induced STAT3 activation seems dispensable for the ABCA1-mediated lipid export. STAT3 represents an intracellular target of IL-10-a cytokine with wellestablished anti-inflammatory properties-and activation of STAT3 was demonstrated to suppress several aspects of inflammatory macrophage activation. These observations raised the interesting possibility that apoA-I might produce anti-inflammatory effects by activating STAT3 in ABCA1-dependent fashion. Actually, exposure of ABCA1-expressing macrophages to apoA-I suppressed lipopolysaccharide (LPS)-induced production of proinflammatory cytokines such as interleukin-6 (IL-6), and this effects were abolished after pharmacological STAT3 blockade or knocking down STAT3 expression with siRNA (Tang et al. 2009). Further investigations demonstrated that the suppressing effect of apoA-I on LPS-induced cytokine production was mediated by mRNA destabilization brought about by mRNA-destabilizing protein tristetraprolin, the expression of which was induced in cells exposed to apoA-I in an ABCA1- and JAK2/STAT3dependent manner (Yin et al. 2011). Recently, apoA-I was demonstrated to promote cyclooxygenase-2 (COX-2) activation and prostacyclin $\left(\mathrm{PGI}_{2}\right)$ production in endothelial cells, and these anti-inflammatory effects were also found to depend on ABCA1 and JAK2 activation (Liu et al. 2011).

Cell division control protein 42 ( $\mathrm{Cdc} 42)$ is a member of a small G-protein family involved in the regulation of cytoskeleton organization and intracellular vesicular trafficking. Early studies revealed decreased Cdc 42 expression and abnormal cytoskeleton architecture in cells obtained from Tangier patients (Hirano et al. 2000). Moreover, impaired cholesterol efflux observed in aged human fibroblasts as well as fibroblasts from Werner syndrome characterized by the early onset of senescent phenotypes including premature atherosclerotic cardiovascular disease could be corrected by forced expression of wild-type Cdc42 (Tsukamoto et al. 2002; Zhang et al. 2005). Expression of constitutively active Cdc42 variant in normal MadinDarby canine kidney (MDCK) cells potentiated apoA-I-induced cholesterol efflux, whereas expression of dominant negative Cdc42 variants in normal MDCK cells or fibroblasts exerted opposite effects (Hirano et al. 2000; Nofer et al. 2003). ApoA-Iinduced cholesterol efflux was also reduced in cells pretreated with Clostridium difficile toxin, which irreversibly modifies and disables Cdc42 (Nofer et al. 2003). Both Cdc42 and ABCA1 were found to co-segregate into lubrol-insoluble, tritonsoluble lipid rafts (Drobnik et al. 2002). In addition, Cdc42 could be immunoprecipitated with wild-type ABCA1, but not with C-terminally truncated variant of this transporter indicating that both proteins interact with each other and suggesting that C-terminus of ABCA1 harbors a potential docking site for Cdc42 (Tsukamoto et al. 2001; Nofer et al. 2006). Exposure of cells expressing wild-type ABCA, but not cells obtained from Tangier patients or expressing C-terminally truncated variant of ABCA1 to apoA-I, induced Cdc42 activation and subsequent actin polymerization (Nofer et al. 2006). Taken together, these findings suggest that apoA-I signals through ABCA1 to activate $\mathrm{Cdc} 42$ and thereby to promote cholesterol efflux. How exactly $\mathrm{Cdc} 42$ activation promotes lipid export from cells remains 
to be elucidated. One possibility is that $\mathrm{Cdc} 42$ modulates intracellular vesicular traffic and thereby helps to supply cholesterol and/or phospholipids to an intracellular compartment, where they are available for the ABCA1-mediated release. The retardation of intracellular lipid transport in cells with reduced Cdc42 levels and its intensification after enforced expression of $\mathrm{Cdc} 42$ appears to support this concept (Tsukamoto et al. 2002). Alternatively, components of the signaling machinery localized downstream to Cdc42 might accelerate intracellular lipid transfer or the ABCA1-dependent lipid translocation across the cell membrane. Actually, apoA-I was demonstrated to produce consecutive activation of two serine/threonine kinases, namely, p21-activated kinase 1 (PAK1) and c-Jun N-terminal kinase (JNK) in ABCA1- and Cdc42-dependent manner (Nofer et al. 2003). The potential involvement of these two kinases in the processes of lipid export from cells has been not clarified to date.

Mobilization of intracellular $\mathrm{Ca}(2+)$ from intracellular stores or $\mathrm{Ca}(2+)$ influx from extracellular space represents an integral component of diverse intracellular signaling pathways. In one single report, evidence has been provided that apoA-I provokes extracellular $\mathrm{Ca}(2+)$ influx in an $\mathrm{ABCA} 1$-dependent manner and that inhibition of calcineurin, the downstream target of $\mathrm{Ca}(2+)$ influx, with cyclosporine A or FK506 completely abolished apoA-I lipidation and also interfered with JAK2 phosphorylation (Karwatsky et al. 2010). However, other authors failed to observe $\mathrm{Ca}(2+)$ mobilization in fibroblasts or smooth muscle cells after exposure to apoA-I or other apolipoproteins (apoA-II, apoC-III) (Nofer et al. 2000 and unpublished observations). Further studies are necessary, therefore, to fully understand the contribution of $\mathrm{Ca}(2+)$ mobilization to $\mathrm{ABCA} 1$-mediated signaling and lipid export.

\section{ApoA-I-Induced Cell Signaling Indirectly Mediated by $\beta$-ATPase and P2Y $_{12 / 13}$ ADP Receptor}

F1F0 ATP synthase is the terminal enzyme of the oxidative phosphorylation pathway responsible for the lion part of ATP synthesis in humans and other living being except in Archaea and present in mitochondria, chloroplasts, and prokaryotic membranes. Unexpectedly, the F1F0 ATP synthase (with F1 domain facing outside) has been found on the surface of several cells including hepatocytes, adipocytes, and endothelial cells, and its $\beta$-chain ( $\beta$-ATPase) has been characterized as a receptor for apoA-I (Martinez et al. 2003b; Fabre et al. 2006; Radojkovic et al. 2009; Howard et al. 2011). The binding of apoA-I to $\beta$-ATPase induces ATP hydrolysis and promotes extracellular ADP generation, which in turn stimulates intracellular signaling by activating purinergic receptors, which belong to the family of G-protein-coupled receptors stimulated by extracellular nucleotides (Martinez et al. 2003b; Fabre et al. 2006). In hepatocytes, the $\beta$-ATPase/P2 $\mathrm{Y}_{13^{-}}$ induced signaling was reported to activate the small G-protein RhoA and the downstream-located Rho-associated protein kinase (ROCK-I) and thereby to promote HDL endocytosis by as yet poorly defined mechanism (Fabre et al. 2006; 
Malaval et al. 2009). In endothelial cells, binding of apoA-I to $\beta$-ATPase and the subsequent generation of extracellular ADP stimulated endothelial proliferation and survival, but components of intracellular signaling cascade responsible for these effects remained uncharacterized (Radojkovic et al. 2009). In a recent study, the activation of $\beta$-ATPase/P2 $\mathrm{Y}_{12}$ was found to precede HDL binding and cell association as well as ABCG1- and SR-BI-dependent internalization, and transcytosis in endothelial cells (Cavelier et al. 2012). The identity of intracellular signaling pathway linking $\mathrm{P}_{2} \mathrm{Y}_{12}$ activation to HDL internalization has not been addressed in this study.

\section{ApoA-I- and HDL-Induced Cell Signaling Indirectly Mediated by ABCA1 and/or ABCG1}

ABCA1 is unique among $\mathrm{ABC}$ transporters in that it requires the direct binding of acceptors for the transported substances. ATP-binding cassette transporter G1 (ABCG1) was also found to be a crucial mediator of lipid export. Studies by Wang et al. demonstrated that ABCG1 promotes efflux to a variety of acceptors, including HDL, low-density lipoprotein (LDL), phospholipid vesicles, and cyclodextrin (Wang et al. 2004, 2006). In major contrast to ABCA1, ABCG1 does not engage into direct interaction with lipid acceptors and for this reason cannot serve as a triggering element of intracellular second messenger cascade. However, recent studies document that ABCG1 (and to lesser extent ABCA1) profoundly impacts on signaling processes taking place in contiguity. Several studies showed that ABCA1 regulates cholesterol distribution between raft and non-raft membrane fractions and pointed to increased lipid raft formation in macrophages and other cells deficient in ABCG1 and ABCA1 (Landry et al. 2006; Sano et al. 2007; Koseki et al. 2007; Zhu et al. 2008a, Yvan-Charvet et al. 2008). Lipid rafts provide a molecular platform securing efficient signal transduction through several receptor families including proinflammatory Toll-like receptors (TLRs). Not surprisingly, therefore, both ABCA1-deficient cells and ABCG1-deficient cells, and in particular cells lacking both ABC transporters, exhibited increased cell surface expression of TLR4 and sensitivity to LPS stimulation (Koseki et al. 2007; Zhu et al. 2008a, Yvan-Charvet et al. 2008; Mogilenko et al. 2012). Also the NF- $\kappa B$ activation-dependent cytokine and chemokine release in response to stimulation with agonists of other TLR receptors (TLR2, TLR3) or other proinflammatory receptors (CD40) revealed to be increased in macrophages deficient in ABCA1 and/or ABCG1 (Sun et al. 2009; Yin et al. 2012). Such cells were also found to be prone to apoptosis during efferocytosis (phagocytosis of apoptotic cells) as compared to wild-type cells (Yvan-Charvet et al. 2010a). The enhanced apoptotic response observed in ABCA1- and ABCG1-deficient macrophages was dependent on an excessive assembly of NADPH oxidase/NOX2 complexes within lipid rafts followed by oxidative burst and ensuing sustained JNK activation, which turned on the apoptotic cell death program. It is very likely that similar mechanisms consisting in amplification of transmembrane signaling under conditions of decreased ABCG1 
and/or ABCA1 activity and the resulting accumulation of lipid rafts may substantially contribute to the enhancement of several proinflammatory processes. For instance, cholesterol accumulation within lipid rafts was suggested to enhance monocyte adhesion and neutrophil activation, and both processes could be reversed by cholesterol removal from cells carried out either with help of synthetic cholesterol acceptors (e.g., cyclodextrin) or through induction of ABCA1-dependent cholesterol efflux (Murphy et al. 2008, 2011). In addition, ABCG1- and ABCA1dependent modulation of cell membrane cholesterol content was demonstrated to critically regulate cellular signaling related to proliferatory responses. For instance, mice deficient in ABCG1- and ABCA1 developed severe monocytosis and neutrophilia, which was dependent on uncontrolled proliferation and expansion of hematopoietic stem progenitor cells (HSPC) (Yvan-Charvet et al. 2010b). The mechanistic analysis revealed increased plasma membrane lipid raft formation in these cells accompanied by elevated cell surface presence of interleukin IL-3/ granulocyte macrophage colony-stimulating factor (GM-CSF) receptor. Addition of IL-3 or GM-CSF to ABCA1/G1-deficient bone marrow led to increased proliferation of HSPCs, reflecting activation of signaling pathways downstream of the IL-3/GM-CSF receptor. Similarly, defects in cholesterol efflux pathways in macrophages and dendritic cells in spleens of ABCA1/G1-deficient mice led to increased production of IL-23 and activation of a proinflammatory and proatherogenic signaling axis involving IL-23/IL-17 (Westerterp et al. 2012). Other studies showed increased lipid raft content in plasma membrane and enhanced proliferation in response to anti-CD3 antibody stimulation in T cells obtained from ABCG1-deficient animals (Bensinger et al. 2008). Similar increase in lymph node T-cell proliferation has been noted after disruption of cholesterol efflux pathway by deletion of apoA-I on a hypercholesterolemic background (Wilhelm et al. 2009).

\section{HDL-Induced Cell Signaling Mediated by SR-BI}

Scavenger receptor type I (SR-BI) is a cell surface glycoprotein originally identified by its homology to the scavenger receptor CD36 and capacity to bind acetylated LDL. Later studies in vitro revealed that SR-BI serves as the binding partner for a variety of ligands including native and oxidized LDL, very-low-density lipoproteins (VLDL), Lp(a), modified serum proteins such as maleylated albumin, lipid vesicles containing negatively charged phospholipids, apoptotic cells, and HDL. Despite this promiscuous binding behavior SR-BI is believed to primarily function as a physiologically relevant HDL receptor because of its tissue expression pattern reflecting sites of HDL cholesterol uptake and because of the profound impact of complete or tissue-specific SR-BI deficiency on HDL metabolism. The classic cellular function of SR-BI is to mediate the selective uptake of lipids from HDL and to transfer them into cells. Cholesteryl esters represent the primary substrate for SR-BI, but this receptor also facilitates transfer of various other lipid species including unesterified cholesterol, phospholipids, triglycerides, and lipid- 
soluble vitamins. SR-BI also mediates the efflux of cholesterol from cells to HDL, which in contrast to ABCA1- and ABCG1-mediated efflux is a passive, concentration gradient-dependent, and energy-independent process. Importantly, SR-BImediated lipid transfer from and to HDL occurs in the absence of consecutive internalization and degradation of HDL particle, which is a precedent condition for effective signal transduction (for recent reviews, see Hoekstra et al. 2010; Mineo and Shaul 2012).

Binding of HDL to SR-BI has been demonstrated to trigger several intracellular signaling events (see Fig. 2). The recruitment of non-receptor tyrosine kinase src to the C-terminal cytoplasmic tail of SR-BI followed by src autophosphorylation and phosphorylation and activation of AMP-activated protein kinase (AMPK) mediated through calcium/calmodulin-dependent protein kinase (CAMK) and/or serine/threonine liver kinase B1 (LKB1) appear to constitute initial steps in SR-BI signaling

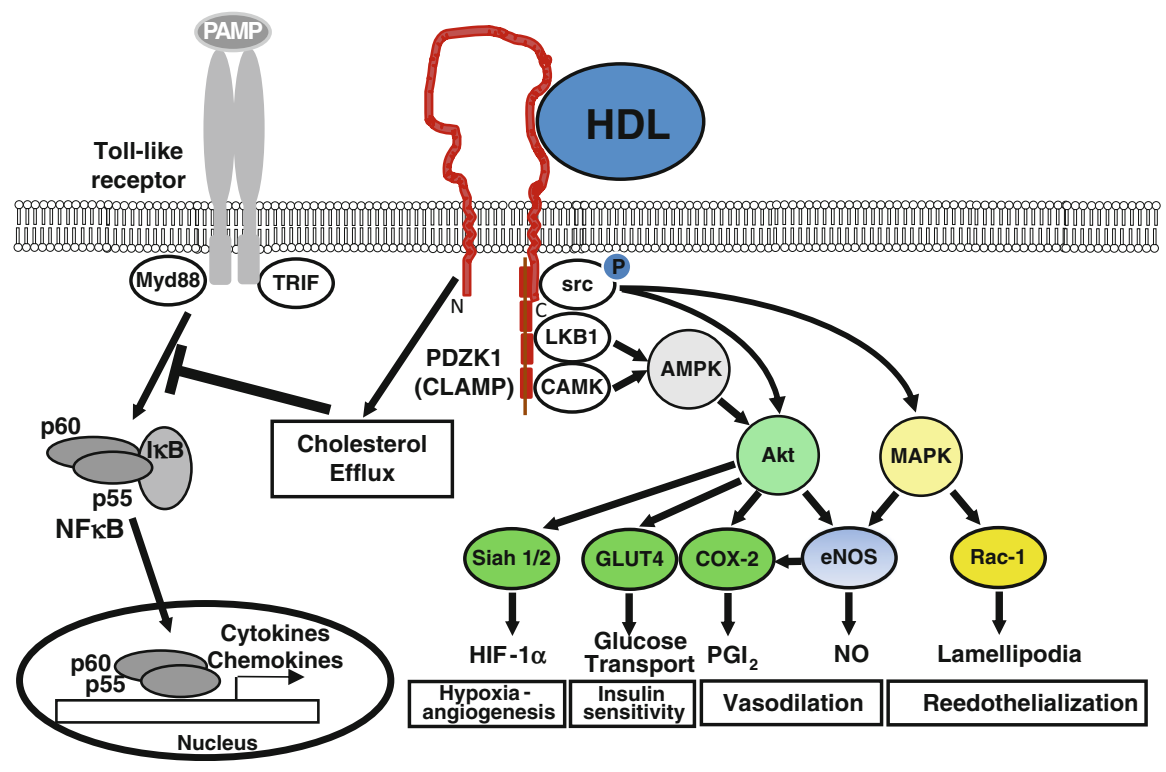

Fig. 2 HDL-induced cell signaling mediated by SR-BI. The binding of HDL to scavenger receptor type 1 (SR-BI) induces activation of protein kinases src, liver kinase B1 (LKB1), and calcium calmodulin-dependent protein kinase (CAMK) in a process dependent on an adapter protein PDZK1 (CLAMP). The resulting activation of AMP-activated protein kinase (AMPK) and protein kinase Akt as well as mitogen-activated protein kinase (MAPK) transduces signal to downstream effectors including endothelial nitric oxide synthase (eNOS) generating NO, cyclooxygenase-2 (COX-2) producing prostacyclin $\left(\mathrm{PGI}_{2}\right)$, glucose transporter GLUT4 mediating cellular glucose uptake, ubiquitin ligases Siah 1 and 2 stabilizing hypoxia-inducible factor-1 $\alpha$ (HIF-1 $\alpha$ ), and small G-protein Rac-1 regulating formation of lamellipodia. These effects account for vasodilatory, insulin-sensitizing, proangiogenic, and endothelial regenerating properties of HDL. In addition, SR-BI-mediated cholesterol efflux to HDL exerts indirect negative effects on signal transduction mediated by proinflammatory Toll-like receptors and the resulting activation of the transcription factor NF- $\mathrm{KB}$ and expression of cyto- and chemokines. Pathogen-associated molecular patterns (PAMP) 
(Mineo et al. 2003; Kimura et al. 2010). Detailed studies revealed that phosphorylations of src (though not its interaction with SR-BI) and AMPK are critically dependent on the presence of the PDZ domain-containing adaptor protein, PDZK1 (also termed CLAMP), which interacts with extreme C-terminal residues of SR-BI and is essential for its stability in the plasma membrane (Kimura et al. 2010; Assanasen et al. 2005; Zhu et al. 2008a, b). Src and AMPK activations lead to the activation of phosphatidylinositol 3-kinase (PI3K), which in turn induces parallel activation of protein kinase B (Akt) and mitogen-activated protein kinase (MAPK) (Kimura et al. 2010; Assanasen et al. 2005; Zhu et al. 2008b). The latter event seems to be required for the subsequent stimulation of the activity of small G-protein Rac1, which promotes formation of lamellipodia and induces cell shape change (Seetharam et al. 2006). It remains currently unclear, whether SRBI-mediated lipid transfer is required for the effective initiation of signaling by HDL. The C-terminal transmembrane domain of SR-BI contains a cholesterolbinding site, which interacts with plasma cholesterol, as could be documented in photocholesterol binding experiments (Assanasen et al. 2005). Studies utilizing SR-BI/CD36 chimeras showed that only transfer of both C-terminal domains (cytoplasmatic and transmembrane) endows CD36 with signaling properties of SR-BI, whereas the transfer of the C-terminal cytoplasmatic domain only is insufficient (Assanasen et al. 2005). In addition, introduction of mutations into the transmembrane C-terminal domain severely impeded SR-BI-mediated signal transduction (Saddar et al. 2013). Other studies pointed to the enhancement of SR-BI signaling in the presence of extracellular cholesterol acceptors such as cyclodextrins or reconstituted HDL particles containing 2 molecules of apoA-I but not cholesterol (Assanasen et al. 2005). Furthermore, HDL enriched in phosphatidylcholine, which increases its cholesterol acceptor capacity, induces signal transduction at concentrations that are otherwise insufficient to invoke signaling. Collectively, these observations suggest that SR-BI-mediated cholesterol efflux exerts at least potentiating effects on HDL-triggered signaling transduced by this receptor. Recently, Al-Jarallah and Trigatti hypothesized that SR-BI-mediated removal of cholesterol from caveolae might suppress the activity of high molecular weight phosphatase complex, which would in turn help to maintain protein kinase targets such as MAPK in the phosphorylated state and to prolong their activation (Al-Jarallah and Trigatti 2010). Whether this mechanism indeed contributes to HDL-induced signaling mediated by SR-BI remains to be clarified.

The concerted activation of both Akt and MAPK appears to constitute the centerpiece of SR-BI-triggered signaling cascades. Both kinases are required for phosphorylation and full activation of endothelial nitric oxide synthase (eNOS), nitric oxide generation, and vasorelaxation, which are observed in endothelial cells or isolated aortas in the presence of HDL (Yuhanna et al. 2001; Mineo et al. 2003; Assanasen et al. 2005). In addition, PI3K/Akt/eNOS signaling was found to be involved in the HDL-induced COX-2 expression and $\mathrm{PGI}_{2}$ release in endothelial cells (Zhang et al. 2012). The proliferation of bone-derived mesenchymal stem cells in response to HDL was likewise mediated via its binding to scavenger receptor-B type I and activation of PI3K/Akt/MAPK pathways (Xu et al. 2012). Activation of 
MAPK but not Akt with subsequent induction of Rac1-GTPase was demonstrated to promote HDL-induced endothelial cell migration, which is critical for carotid artery reendothelialization after perivascular electric injury (Seetharam et al. 2006). Conversely, activation of Akt but not MAPK was necessary to sustain the migration and the proliferation of breast cancer cells (Danilo et al. 2013). In adipocytes, SRBI-mediated activations of Akt and AMPK were found to promote glucose uptake and translocation of the glucose transporter GLUT4 to the plasma membrane suggesting that SR-BI signaling might contribute to insulin-sensitizing effects of HDL (Zhang et al. 2011). Finally, augmentation of hypoxia-induced angiogenesis, which is observed in the presence of HDL, was demonstrated to depend on SR-BImediated activation of Akt linked to increased expression of E3 ubiquitin ligases Siah1 and Siah2 and accelerated degradation of prolyl hydroxylases (PHD1-PHD3) combined with stabilization of hypoxia-inducible factor $1 \alpha$ (HIF-1 $\alpha$ ) (Tan et al. 2013). Recent studies provide evidence that similar to ABCG1 also SR-BI may indirectly affect proinflammatory signaling by reducing membrane cholesterol content. Actually, macrophages obtained from SR-BI-deficient animals were characterized by increased inflammatory responses to Toll-like receptor agonists (Guo et al. 2009; Cai et al. 2012). Feng et al. found that SR-BI deficiency enhanced lymphocyte proliferation, caused imbalanced interferon- $\gamma$ (IFN $\gamma$ ) and IL-4 production in lymphocytes, and led to elevated inflammatory cytokine production in macrophages (Feng et al. 2011). Umemoto et al. demonstrated that HDL-induced disruption of lipid raft in adipocytes, which is accompanied by reduced activation of NADPH oxidase and production of monocyte chemotactic protein 1 (MCP1), was reversed by silencing both ABCG1 and SR-BI expression (Umemoto et al. 2013).

It is well established that HDL inhibits the activation of platelets by strong agonists such as thrombin and collagen (for review, see Nofer and van Eck 2011). The presence of SR-BI has been identified on platelet surface (Imachi et al. 2003). Recent investigations demonstrated that HDL binding to platelets was reduced in a concentration-dependent fashion by SR-BI ligands such as negatively charged liposomes, which in addition potently inhibited thrombin-induced platelet aggregation, granule secretion, fibrinogen binding, and mobilization of intracellular $\mathrm{Ca}^{2+}$ (Nofer et al. 2011). Furthermore, both native HDL and other SR-BI ligands failed to inhibit thrombin-induced activation of platelets obtained from SR-BI-deficient mice. These findings together with the results of Imachi et al. (2003), who observed an inverse relationship between the levels of SR-BI expression and the platelet aggregation in response to ADP, corroborate the hypothesis that SR-BI is a true functional HDL receptor on platelets. The likely consequence of the HDL binding to SR-BI on platelets is the induction of intracellular second messenger cascade. It has been previously demonstrated that incubation of platelets with HDL leads to the release of DAG from the plasma membrane phosphatidylcholine and to subsequent activation of $\mathrm{PKC}$, which in turn stimulates the $\mathrm{Na}^{+} / \mathrm{H}^{+}$antiport, promotes the alkalization of the cytoplasmic compartment, and inhibits the release of calcium ions from intracellular storage sites (Nofer et al. 1996). In addition, activation of PKC inhibits the activity of phosphatidylinositol-specific phospholipase C (PI-PLC), which is one of 
the most important signal transduction mediators of agonists such as thrombin and collagen (Nofer et al. 1998). It is conceivable that this signaling cascade is launched by interaction of HDL with SR-BI on platelet surface.

\section{HDL-Induced Cell Signaling Mediated by S1P}

Early studies on HDL-induced signal transduction focused on apoA-I or other apolipoproteins as potential binding partners for HDL receptor responsible for initiating the cascade of intracellular signaling events. However, it has soon become clear that neither apoA-I nor other proteins encountered in HDL particles can exclusively account for HDL signaling activities. Studies utilizing fibroblasts demonstrated that PI-PLC activation, which is observed in cells exposed to HDL and accompanied by increased phosphatidylinositol bisphosphate $\left(\mathrm{PIP}_{2}\right)$ turnover and the mobilization of intracellular calcium, is not emulated by purified apoA-I or apoA-I-containing proteoliposomes, but occurs in the presence of lipids extracted from HDL holoparticles (Nofer et al. 2000). Likewise, certain cellular responses triggered by HDL in fibroblasts of smooth muscle cells such as cell proliferation or proliferation-linked activation of MAPK could be observed in the presence of HDL lipids or HDL particles, in which proteins were covalently modified or enzymatically degraded, but not in the presence of proteins isolated from HDL (Deeg et al. 1997; Sachinidis et al. 1999; Nofer et al. 2001a). The intense search for lipid species accounting for the signal-inducing capacity of HDL revealed that these lipoproteins serve as a carrier for bioactive lysosphingolipids such as sphingosine-1-phosphate (S1P), sphingosylphosphorylcholine (SPC), and lysosulfatide (LSF) and that signaling events observed in cells exposed to native HDL or isolated lysosphingolipids largely overlap (Sachinidis et al. 1999; Nofer et al. 2000, 2004; Kimura et al. 2001).

$\mathrm{S} 1 \mathrm{P}$ is the most prominent and best characterized member of the lysosphingolipid family. It is produced by phosphorylation of sphingosine by sphingosine kinases 1 and 2 (SK1 and SK2) in response to a variety of stimuli and degraded to phosphoethanolamine and hexadecanal by S1P-lyase or to sphingosine and organic phosphate by S1P phosphatase. S1P interacts with five related G-protein-coupled

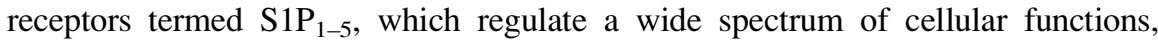
including proliferation and survival, cytoskeletal rearrangements, and cell motility, and exert potent cytoprotective effects. In the vasculature, S1P receptors were identified on endothelial and smooth muscle cells, and S1P provides essential contribution to the new vessel formation and the maintenance of vascular barrier integrity. In addition, S1P was demonstrated to interfere with proliferation, migration, and activation of monocytes/macrophages and to regulate their recruitment to sites of inflammation (for recent reviews, see Maceyka et al. 2012; Levkau 2013). Erythrocytes and platelets are major sources of S1P in plasma, where it is preferentially (up to $60 \%$ ) associated with HDL subfraction rich in apolipoprotein M (apoM) (Dahm et al. 2006; Hänel et al. 2007; Christoffersen et al. 2011). Recent studies demonstrate that apoM specifically binds S1P and stimulates its biosynthesis for secretion (Liu et al. 2013b). There is substantial evidence suggesting that S1P may 
directly contribute to atheroprotective effects attributed to HDL. For instance, S1P closely correlates with HDL in a concentration range, in which HDL most effectively protects against atherosclerosis, and decreased HDL-bound S1P levels were noted in patients with coronary artery disease and myocardial infarction (Karuna et al. 2011; Sattler et al. 2010; Argraves et al. 2011). In addition, S1P was found to emulate in vitro several atheroprotective effects of HDL, which themselves could be attenuated by interrupting signaling through S1P receptors (Levkau et al. 2013).

Protein kinases MAPK, AMPK, and Akt are critical for the S1P-dependent signaling cascade, which unfolds in endothelial cells in the presence of HDL (see Fig. 3 for schematic presentation of S1P-mediated signaling). Kimura and

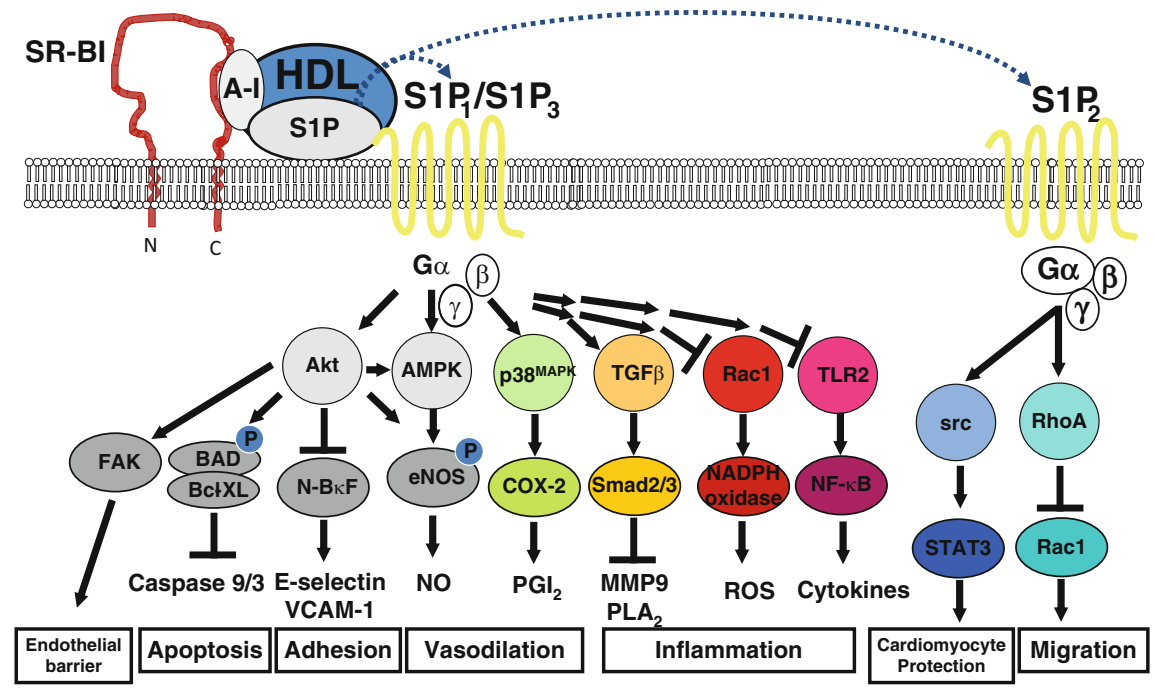

Fig. 3 HDL-induced cell signaling mediated by S1P. The binding of HDL to scavenger receptor type I (SR-BI) may provide a molecular platform for efficient receptor stimulation by sphingosine1-phosphate (S1P) present in HDL molecule. Activation of AMP-activated protein kinase (AMPK) and protein kinase Akt via $\mathrm{S}_{1} \mathrm{P}_{1}$ and/or $\mathrm{S}_{1} \mathrm{P}_{3}$ receptors leads to the stimulation of focal adhesion kinase (FAK), the inactivation of proapoptotic protein BAD and the inhibition of caspases 9 and 3, the inhibition of the transcription factor NF- $\mathrm{KB}$ and the expression of adhesive molecules E-selectin and vascular cell adhesion molecule 1 (VCAM-1), as well as the activation of endothelial nitric oxide synthase (eNOS) and the generation of NO. Activation of the mitogenactivated protein kinase p38MAPK results in activation of cyclooxygenase-2 (COX-2) and production of prostacyclin $\left(\mathrm{PGI}_{2}\right)$. Increased expression of transforming growth factor $\beta$ (TGF $\beta$ ) contributes to the increased activation of transcription factors Smad 2 and 3, which suppress production of proinflammatory matrix metalloproteinases such as MMP9 as well as phospholipase $\mathrm{A}_{2}\left(\mathrm{PLA}_{2}\right)$. Inhibition of small G-protein Rac1 suppresses activity of NADPH oxidase and generation of reactive oxygen species. Inhibition of Toll-like receptor 2 (TLR2) reduces NF- $\mathrm{KB}$ activation and production of proinflammatory cytokines. These effects account for endothelial barrier-enhancing, anti-inflammatory, antiapoptotic, anti-adhesive, and vasodilatory properties of HDL. Activation of protein kinase src and transcription factor signal transducer and activator of transcription 3 (STAT3) via $\mathrm{S}_{2} \mathrm{P}_{2}$ protects against cardiomyocyte apoptosis. Activation of the small G-protein RhoA via $\mathrm{S}_{1} \mathrm{P}_{2}$ leads to inhibition of the small G-protein Rac1, which regulates smooth muscle cell migration 
colleagues demonstrated that activation of MAPK is essential for endothelial proliferation and survival mediated by HDL-associated lysosphingolipids (Kimura et al. 2001). Using antisense oligonucleotides and siRNA strategy combined with application of pharmacological G-protein inhibitors, they further show that S1P receptor types 1 and 3 and inhibitory G-protein (Gi) contribute to HDL-mediated induction of endothelial migration, whereas the augmentation of endothelial survival in endothelial cells exposed to HDL is exclusively dependent on $\mathrm{S}_{1} \mathrm{P}_{1}$. Miura et al. demonstrated that HDL promotes endothelial cell tube formation via activation of MAPK and suggested that this effect is mediated by S1P present in HDL particles (Miura et al. 2003). Later these authors showed that reconstituted HDL particles enriched in S1P are particularly effective in stimulating angiogenic response (Matsuo et al. 2007). HDL-like lipoproteins are also present in follicular fluid and induce angiogenesis in the S1P- and MAPK-dependent manner (von Otte et al. 2006). The activation of AMPK, which itself was entirely dependent on the parallel activation of CAMK, was localized upstream to Akt in the signaling sequel (Kimura et al. 2010). The stimulation of Akt by HDL-associated lysosphingolipids was - depending on the experimental system used-mediated by either $\mathrm{S}_{1} \mathrm{P}_{1}$ or $\mathrm{S} \mathrm{P}_{3}$ and controlled an array of important antiatherogenic activities attributed to HDL. Nofer et al. (2001b) found that activation of Akt by HDL and its associated lysosphingolipids disabled a proapoptotic protein BAD and thereby inhibited the collapse of mitochondrial potential, the cytochrome $\mathrm{C}$ release from mitochondria, and the ensuing activation of apoptosis-executing caspases 3 and 9. Nofer et al. (2004) and Kimura et al. (2006) demonstrated that the S1P content in HDL at least partially accounts for the HDL-stimulated activation of eNOS, generation of NO, and vasorelaxation. Stimulation of endothelial cells with statins such as pitavastatin or simvaststin increased the expression of $\mathrm{S}_{1} \mathrm{P}_{1}$ and potentiated the stimulatory effect of HDL on eNOS activation (Igarashi et al. 2007; Kimura et al. 2008). Likewise, upregulated $\mathrm{S}_{1} \mathrm{P}_{1}$ and $\mathrm{S}_{1} \mathrm{P}_{3}$ expression and increased Akt and eNOS activation were found in fenofibrate-treated mice, which were characterized by elevated plasma levels of HDL and S1P (Krishna et al. 2012). Kimura et al. (2006) reported that signaling through $\mathrm{S}_{1} \mathrm{P}_{1}$ receptor partly accounts for the inhibitory effects exerted by HDL on tumor necrosis factor $\alpha$ (TNF $\alpha$ )induced NF- $\mathrm{kB}$ activation as well as endothelial expression of adhesins such as vascular cell adhesion molecule-1 (VCAM-1) and intercellular adhesion molecule1 (ICAM-1). Argraves et al. (2008) found that HDL increased endothelial barrier integrity as measured by electric cell substrate impedance sensing in a process that was entirely dependent on $\mathrm{S} 1 \mathrm{P}, \mathrm{S}_{1} \mathrm{P}_{1}$ expression, and Akt activation. The favorable effect of S1P on endothelial barrier was sustained for considerably longer time with HDL-associated rather than albumin-associated S1P, and this was related to the inhibitory effect of HDL on $\mathrm{S}_{1} \mathrm{P}_{1}$ degradation (Wilkerson et al. 2012). Norata et al. reported that HDL potently stimulates endothelial expression of long pentraxin 3-a key component of innate immunity-as well as transforming growth factor- $\beta$ (TGF $\beta$ ), a compound with potent anti-inflammatory and immunomodulatory activities, in Akt-dependent manner. They attributed these effects to the presence of S1P and SPC in HDL particles and $\mathrm{S}_{1} \mathrm{P}_{1}$ and $\mathrm{S}_{1} \mathrm{P}_{3}$ receptors on 
endothelial cells (Norata et al. 2005, 2008). Increased activation of Smad 2/3, a transcription factor controlled by TGF $\beta$, found in the latter study suggests that HDL-associated S1P may transactivate TGF $\beta$ signaling pathways in an autocrine fashion. Most recently, Tatematsu et al. reported that endothelial lipase (EL) is a critical determinant of HDL-stimulated S1P-dependent signaling (Tatematsu et al. 2013). Actually, HDL-induced endothelial cell migration and angiogenic responses as well as Akt activation were markedly decreased in EL-deficient endothelial cells, but restored in the presence of exogenous S1P. Basing on application of pharmacological inhibitors, Tatematsu and colleagues concluded that the effects of HDL on endothelial cell migration and Akt activation are mediated by $\mathrm{S}_{1} \mathrm{P}_{1}$.

As pointed out above, HDL is strongly mitogenic for smooth muscle cells, and HDL-associated lysosphingolipids appear to account for this effect. On the other hand, HDL was identified as a potent inhibitor of smooth muscle cell migration, which is a hallmark of the development of advanced atherosclerotic plaque and restenosis (Tamama et al. 2005; Damirin et al. 2007). The inhibitory effect of HDL was likely mediated by its S1P content, as it was abolished in the presence of $\mathrm{S}_{1} \mathrm{P}_{2^{-}}$ specific siRNA and following desensitization of S1P receptors and much enhanced in cells overexpressing $\mathrm{S}_{\mathrm{P}} \mathrm{P}_{2}$ receptor. Further effects exerted by HDL on smooth muscle cell through its S1P content include modulation of the activity of several vasoactive factors. Chrisman et al. found that HDL-associated S1P desensitizes guanylyl cyclase $\mathrm{B}$, a receptor for C-type natriuretic peptide (CNP), and thereby inhibits CNP-induced cyclic guanosine monophosphate (cGMP) accumulation in vascular smooth muscle cells (Chrisman et al. 2003). Gonzalez-Diez and colleagues demonstrated induction of $\mathrm{COX}-2$ expression and $\mathrm{PGI}_{2}$ production in vascular smooth muscle cells exposed to HDL, which was mediated through S1P receptors 2 and 3 and protein kinase p38MAPK (González-Díez et al. 2008). They have also shown that simvastatin potentiates the HDL- and S1P-induced COX-2 expression by upregulating cellular levels of $\mathrm{S}_{1} \mathrm{P}_{3}$. Finally, HDL-associated lysosphingolipids S1P and SPC were found to suppress the proinflammatory activation of smooth muscle cells brought about by stimulation with thrombin and characterized by the increased release of inflammatory cyto- and chemokines such as MCP1 (Tölle et al. 2008). The detailed examination of signaling pathways accounting for inhibitory effects exerted by HDL-associated lysosphingolipids revealed that they were related to the inhibition of Rac1-mediated activation of NADPH oxidase and generation of reactive oxygen species. $\mathrm{S}_{1} \mathrm{P}_{3}$ was identified to mediate antiinflammatory effects of HDL, S1P, and SPC in smooth muscle cells.

Cardiomyocytes express several S1P receptors, and HDL was found to exert protective effects on these cells through its S1P content. HDL and S1P showed potent capacity to protect cardiomyocytes against hypoxia-reoxygenation- or doxorubicin-induced apoptosis, and these effects were attributed to the induction of protein kinases Src, MAPK, and Akt and the transcription factor STAT3 (Frias et al. 2009, 2010; Tao et al. 2010). However, investigations concerning the identity of receptor-mediating protective effects of HDL-associated S1P were inconclusive, and equally supportive evidence for the involvement of either $\mathrm{S}_{1} \mathrm{P}_{2}$ 
or $\mathrm{S}_{1} \mathrm{P}_{1}$ and $\mathrm{S}_{1} \mathrm{P}_{3}$ has been provided. Recently, short-term treatment of cardiomyocytes with HDL or S1P was found to induce a PKC-dependent phosphorylation of connexin43, which is a gap junction protein present in ventricular cardiomyocytes and involved in cardioprotection by modulating ischemic preconditioning (Morel et al. 2012). In addition, HDL and S1P improved gap junctional communication, but only incrementally affected conduction velocities in cardiomyocytes. The physiological relevance of these effects has been clearly documented using an ex vivo approach, in which both HDL and S1P protected perfused hearts against ischemia/reperfusion-induced cell death.

Several other cell types were found to be responsive to stimulation with HDL-associated lysosphingolipids, but the physiological relevance of these effects remains obscure. For instance, HDL and S1P were demonstrated to stimulate prostate cancer cell migration and invasion through S1P receptors 2 and 3 and activation of MAPK and STAT3, to stimulate plasminogen activator inhibitor-1 (PAI-1) release from adipocytes through $\mathrm{S}_{2} \mathrm{P}_{2}$ and activation of $\mathrm{PKC}$ and Rho kinase, and to promote release of fibroblast growth factor-2 (FGF2), a potent neurotrophic factor, from astroglial cells through activation of PI-PLC and MAPK (Malchinkhuu et al. 2003; Lee et al. 2010; Sekine et al. 2011). HDL particles isolated from body fluids other than plasma were also found to contain S1P and to stimulate intracellular signaling through their S1P content. For instance, HDL-like particles obtained from cerebrospinal fluid stimulated astrocyte migration and oligodendrocyte retraction in a manner sensitive to inhibitors of S1P receptors 1 and 3 (Sato et al. 2007). Follicular fluid-derived HDL was found to promote human granulosa lutein cell migration through $\mathrm{S}_{3} \mathrm{P}_{3}$ and activation of small G-protein Rac1 (Becker et al. 2011). Despite the prominent role played by monocytes and macrophages in the pathogenesis of atherosclerosis, very little is known about the modulatory effects exerted by S1P cargo of HDL particles on these cells. In one study, both HDL and S1P were found to blunt monocyte activation induced by agonists of TLR2, but the attribution of this effect to the HDL-associated S1P was not unequivocally demonstrated (Dueñas et al. 2008).

\section{HDL-Induced Cell Signaling: Future Challenges and Opportunities}

HDL constitutes a large and heterogenous macromolecule carrying more than 50 proteins, several amphipathic peptides, miRNAs, and hundreds of lipid species representing almost all known lipid classes. Several proteins and lipids identified in HDL_-among others, apolipoprotein H, haptoglobin, and steroid hormones - are recognized receptor ligands and inductors of intracellular signaling events. However, the rigid evidence demonstrating that these proteins or lipids contribute to the HDL-induced signaling governing diverse functional responses to this lipoprotein has been not provided to date or is at best equivocal. For instance, several studies documented that HDL carries estradiol in the form of fatty acyl esters produced in a reaction catalyzed by LCAT. HDL-derived estradiol esters are internalized via SR- 
BI-mediated mechanisms and subsequently hydrolyzed intracellularly to unfold their effects. However, the specific signaling events and/or physiological effects clearly attributable to the estradiol content in HDL particles have not been unequivocally defined. In one study, HDL-associated estradiol was found to stimulate eNOS and vasodilation in an SR-BI- and Akt-dependent manner, but these results were questioned by others (Gong et al. 2003; Nofer et al. 2004). In other study, dehydroepiandrosterone (DHEA) fatty acyl esters once incorporated in HDL were found to induce stronger vasodilatory response, and this effect appeared also to be mediated by SR-BI (Paatela et al. 2011). However, no clear signaling link between the HDL-associated DHEA and vasodilation has been established in this study. These two examples clearly show that the assignment of ligands present in HDL to the appending signaling machinery and to various functions exerted by these lipoproteins in physiology will be a major challenge in the future.

The composition of HDL particles is variable and influenced both by normal metabolism and in various states of pathology. For instance, HDL particles isolated from subjects suffering from acute or chronic inflammatory diseases were found to lose proteins and enzymes with established or presumed antiatherogenic function such as apoA-I or LCAT and to concomitantly acquire proinflammatory or prooxidative factors such as serum amyloid A (SAA), ceruloplasmin, lipoproteinspecific phospholipase $A_{2}$, or myeloperoxidase. Not surprisingly, such inflammatory HDL particles are severely impeded in their capacity to trigger certain signaling pathways, but at the same time may acquire new ligands and thereby alter their signaling properties. For instance, the relatively high content of apolipoprotein $\mathbf{J}$ (apoJ) in HDL particles obtained from healthy subjects endowed them with important antiatherogenic property to promote endothelial survival by stimulation of the expression of antiapoptotic protein Bcl-XL (Riwanto et al. 2013). By contrast, HDL particles isolated from patients with stable coronary heart disease (CHD) or acute coronary syndromes were found to be poor in apoJ, but instead enriched in apoC-I or apoC-III (McNeal et al. 2013; Riwanto et al. 2013). Furthermore, the increased content of these apolipoproteins turned HDL particles into potent apoptosis inducers in vascular smooth muscle and endothelial cells by virtue of stimulating the expression of proapoptotic protein tBid. In addition to CHD, dysfunctional HDL is also encountered in chronic conditions such as chronic kidney disease (CKD). Abnormal HDL composition characterized primarily by increased serum amyloid A and apoC-III content as well as compromised HDL functionality has been repeatedly reported in uremic patients (Holzer et al. 2011; Weichhart et al. 2012). In one recent study, SAA-enriched HDL encountered in CKD was found to induce production of a proinflammatory chemokine MCP1 in smooth muscle cells by interaction with formyl peptide receptor 2 (FPR2) (Tölle et al. 2012). In other study conducted in CKD patients, accumulation of symmetric dimethylarginine (SDMA) in HDL was found to turn this lipoprotein into a noxious particle reducing NO bioavailability, evoking endothelial dysfunction, and subsequently increasing arterial blood pressure. The deleterious effects of SDMA-modified HDL were attributed to the noncanonical activation of TLR2 (Speer et al. 2013). This exemplifies another challenge facing HDL research in the future, namely, 
identification of ligands, receptors, and signaling cascades attributable to HDL in various states of pathology.

Notwithstanding the physiological or pathological character of HDL-induced signal transduction, its elucidation may open new opportunities for the development of antiatherogenic, anti-inflammatory, or antithrombotic drugs. Several interventional studies conducted recently and aiming at cardiovascular risk reduction through simple elevation of plasma HDL cholesterol fall through. This epic failure emphasizes the necessity to develop more sophisticated strategies based on targeted exploitation of selected antiatherogenic or anti-inflammatory activities exerted by HDL particles. The proper understanding of HDL-induced signaling may help to specifically enhance beneficial physiological or to disable harmful pathological effects of HDL and thereby to design more efficacious therapeutic approaches for cardiovascular and other inflammatory diseases.

Open Access This chapter is distributed under the terms of the Creative Commons Attribution Noncommercial License, which permits any noncommercial use, distribution, and reproduction in any medium, provided the original author(s) and source are credited.

\section{References}

Al-Jarallah A, Trigatti BL (2010) A role for the scavenger receptor, class B type I in high density lipoprotein dependent activation of cellular signaling pathways. Biochim Biophys Acta 1801:1239-1248

Arakawa R, Yokoyama S (2002) Helical apolipoproteins stabilize ATP-binding cassette transporter A1 by protecting it from thiol protease-mediated degradation. J Biol Chem 277:2242622429

Argraves KM, Gazzolo PJ, Groh EM et al (2008) High density lipoprotein-associated sphingosine 1-phosphate promotes endothelial barrier function. J Biol Chem 283:25074-25081

Argraves KM, Sethi AA, Gazzolo PJ et al (2011) S1P, dihydro-S1P and C24:1-ceramide levels in the HDL-containing fraction of serum inversely correlate with occurrence of ischemic heart disease. Lipids Health Dis 10:70

Assanasen C, Mineo C, Seetharam D et al (2005) Cholesterol binding, efflux, and a PDZ-interacting domain of scavenger receptor-BI mediate HDL-initiated signaling. J Clin Invest 115:969-977

Becker S, von Otte S, Robenek H et al (2011) Follicular fluid high-density lipoprotein-associated sphingosine 1-phosphate (S1P) promotes human granulosa lutein cell migration via S1P receptor type 3 and small G-protein RAC1. Biol Reprod 84:604-612

Bensinger SJ, Bradley MN, Joseph SB et al (2008) LXR signaling couples sterol metabolism to proliferation in the acquired immune response. Cell 134:97-111

Bodzioch M, Orso E, Klucken J et al (1999) The gene encoding ATP-binding cassette transporter 1 is mutated in Tangier disease. Nat Genet 22:347-351

Brooks-Wilson A, Marcil M, Clee SM et al (1999) Mutations in ABC1 in Tangier disease and familial high-density lipoprotein deficiency. Nat Genet 22:336-345

Cai L, Wang Z, Meyer JM et al (2012) Macrophage SR-BI regulates LPS-induced pro-inflammatory signaling in mice and isolated macrophages. J Lipid Res 53:1472-1481

Cavelier C, Ohnsorg PM, Rohrer L et al (2012) The $\beta$-chain of cell surface F(0)F(1) ATPase modulates apoA-I and HDL transcytosis through aortic endothelial cells. Arterioscler Thromb Vasc Biol 32:131-139 
Chrisman TD, Perkins DT, Garbers DL (2003) Identification of a potent serum factor that causes desensitization of the receptor for C-Type natriuretic peptide. Cell Commun Signal 1:4

Christoffersen C, Obinata H, Kumaraswamy SB et al (2011) Endothelium-protective sphingosine1-phosphate provided by HDL-associated apolipoprotein M. Proc Natl Acad Sci USA 108:9613-9618

Chroni A, Liu T, Fitzgerald ML, Freeman MW et al (2004) Cross-linking and lipid efflux properties of apoA-I mutants suggest direct association between apoA-I helices and ABCA1. Biochemistry 43:2126-2139

Dahm F, Nocito A, Bielawska A et al (2006) Distribution and dynamic changes of sphingolipids in blood in response to platelet activation. J Thromb Haemost 4:2704-2709

Damirin A, Tomura H, Komachi M et al (2007) Role of lipoprotein-associated lysophospholipids in migratory activity of coronary artery smooth muscle cells. Am J Physiol Heart Circ Physiol 292:H2513-H2522

Danilo C, Gutierrez-Pajares JL et al (2013) Scavenger receptor class B type I regulates cellular cholesterol metabolism and cell signaling associated with breast cancer development. Breast Cancer Res 15:R87

Deeg MA, Bowen RF, Oram JF et al (1997) High density lipoproteins stimulate mitogen-activated protein kinases in human skin fibroblasts. Arterioscler Thromb Vasc Biol 17:1667-1674

Drobnik W, Borsukova H, Böttcher A et al (2002) Apo AI/ABCA1-dependent and HDL3mediated lipid efflux from compositionally distinct cholesterol-based microdomains. Traffic 3:268-278

Dueñas AI, Aceves M, Fernández-Pisonero I et al (2008) Selective attenuation of Toll-like receptor 2 signalling may explain the atheroprotective effect of sphingosine 1-phosphate. Cardiovasc Res 79:537-544

Fabre AC, Vantourout P, Champagne E et al (2006) Cell surface adenylate kinase activity regulates the $\mathrm{F}(1)$-ATPase/P2Y (13)-mediated HDL endocytosis pathway on human hepatocytes. Cell Mol Life Sci 63:2829-2837

Feng H, Guo L, Wang D et al (2011) Deficiency of scavenger receptor BI leads to impaired lymphocyte homeostasis and autoimmune disorders in mice. Arterioscler Thromb Vasc Biol 31:2543-2551

Fitzgerald ML, Morris AL, Rhee JS et al (2002) Naturally occurring mutations in the largest extracellular loops of ABCA1 can disrupt its direct interaction with apolipoprotein A-I. J Biol Chem 277:33178-33187

Fitzgerald ML, Morris AL, Chroni A et al (2004) ABCA1 and amphipathic apolipoproteins form high-affinity molecular complexes required for cholesterol efflux. J Lipid Res 45:287-294

Francis GA, Knopp RH, Oram JF (1995) Defective removal of cellular cholesterol and phospholipids by apolipoprotein A-I in Tangier disease. J Clin Invest 96:78-87

Frias MA, James RW, Gerber-Wicht C et al (2009) Native and reconstituted HDL activate Stat 3 in ventricular cardiomyocytes via ERK1/2: role of sphingosine-1-phosphate. Cardiovasc Res 82:313-323

Frias MA, Lang U, Gerber-Wicht $\mathrm{C}$ et al (2010) Native and reconstituted HDL protect cardiomyocytes from doxorubicin-induced apoptosis. Cardiovasc Res 85:118-126

Glomset JA (1968) The plasma lecithin: cholesterol acyltransferase reaction. J Lipid Res 9:155167

Gong M, Wilson M, Kelly T et al (2003) HDL-associated estradiol stimulates endothelial NO synthase and vasodilation in an SR-BI-dependent manner. J Clin Invest 111:1579-1587

González-Díez M, Rodríguez C, Badimon L et al (2008) Prostacyclin induction by high-density lipoprotein (HDL) in vascular smooth muscle cells depends on sphingosine 1-phosphate receptors: effect of simvastatin. Thromb Haemost 100:119-126

Graham DL, Oram JF (1987) Identification and characterization of a high density lipoproteinbinding protein in cell membranes by ligand blotting. J Biol Chem 262:7439-7442

Guo L, Song Z, Li M, Li XA et al (2009) Scavenger receptor BI protects against septic death through Its role in modulating inflammatory response. J Biol Chem 284:19826-19834 
Haidar B, Mott S, Boucher B et al (2001) Cellular cholesterol efflux is modulated by phospholipidderived signaling molecules in familial HDL deficiency/Tangier disease fibroblasts. J Lipid Res 42:249-257

Haidar B, Denis M, Krimbou L et al (2002) cAMP induces ABCA1 phosphorylation activity and promotes cholesterol efflux from fibroblasts. J Lipid Res 43:2087-2094

Haidar B, Denis M, Marcil M et al (2004) Apolipoprotein A-I activates cellular cAMP signaling through the ABCA1 transporter. J Biol Chem 279:9963-9969

Hänel P, Andréani P, Gräler MH (2007) Erythrocytes store and release sphingosine 1-phosphate in blood. FASEB J 21:1202-1209

Hirano K, Matsuura F, Tsukamoto K et al (2000) Decreased expression of a member of the Rho GTPase family, Cdc $42 \mathrm{Hs}$, in cells from Tangier disease - the small $\mathrm{G}$ protein may play a role in cholesterol efflux. FEBS Lett 484:275-279

Hoekstra M, Van Berkel TJ, Van Eck M (2010) Scavenger receptor BI: a multi-purpose player in cholesterol and steroid metabolism. World J Gastroenterol 16:5916-5924

Holzer M, Birner-Gruenberger R, Stojakovic T et al (2011) Uremia alters HDL composition and function. J Am Soc Nephrol 22:1631-1641

Howard AD, Verghese PB, Arrese EL et al (2011) The $\beta$-subunit of ATP synthase is involved in cellular uptake and resecretion of apoA-I but does not control apoA-I-induced lipid efflux in adipocytes. Mol Cell Biochem 348:155-164

Hu YW, Ma X, Li XX et al (2009) Eicosapentaenoic acid reduces ABCA1 serine phosphorylation and impairs ABCA1-dependent cholesterol efflux through cyclic AMP/protein kinase A signaling pathway in THP-1 macrophage-derived foam cells. Atherosclerosis 204:e35-e43

Igarashi J, Miyoshi M, Hashimoto T et al (2007) Statins induce S1P1 receptors and enhance endothelial nitric oxide production in response to high-density lipoproteins. Br J Pharmacol 150:470-479

Imachi H, Murao K, Cao W et al (2003) Expression of human scavenger receptor B1 on and in human platelets. Arterioscler Thromb Vasc Biol 23:898-904

Iwamoto N, Lu R, Tanaka N, Abe-Dohmae S et al (2010) Calmodulin interacts with ATP binding cassette transporter A1 to protect from calpain-mediated degradation and upregulates highdensity lipoprotein generation. Arterioscler Thromb Vasc Biol 30:1446-1452

Karuna R, Park R, Othman A et al (2011) Plasma levels of sphingosine-1-phosphate and apolipoprotein $\mathrm{M}$ in patients with monogenic disorders of HDL metabolism. Atherosclerosis 219:855863

Karwatsky J, Ma L, Dong F et al (2010) Cholesterol efflux to apoA-I in ABCA1-expressing cells is regulated by $\mathrm{Ca} 2$ +-dependent calcineurin signaling. J Lipid Res 51:1144-1156

Kimura T, Sato K, Kuwabara A et al (2001) Sphingosine 1-phosphate may be a major component of plasma lipoproteins responsible for the cytoprotective actions in human umbilical vein endothelial cells. J Biol Chem 276:31780-31785

Kimura T, Tomura H, Mogi C et al (2006) Role of scavenger receptor class B type I and sphingosine 1-phosphate receptors in high density lipoprotein-induced inhibition of adhesion molecule expression in endothelial cells. J Biol Chem 281:37457-37467

Kimura T, Mogi C, Tomura H et al (2008) Induction of scavenger receptor class B type I is critical for simvastatin enhancement of high-density lipoprotein-induced anti-inflammatory actions in endothelial cells. J Immunol 181:7332-7340

Kimura T, Tomura H, Sato K et al (2010) Mechanism and role of high density lipoprotein-induced activation of AMP-activated protein kinase in endothelial cells. J Biol Chem 285:4387-4397

Koseki M, Hirano K, Masuda D et al (2007) Increased lipid rafts and accelerated lipopolysaccharide-induced tumor necrosis factor-alpha secretion in Abca1-deficient macrophages. J Lipid Res 48:299-306

Krishna SM, Seto SW, Moxon JV et al (2012) Fenofibrate increases high-density lipoprotein and sphingosine 1 phosphate concentrations limiting abdominal aortic aneurysm progression in a mouse model. Am J Pathol 181:706-718 
Landry YD, Denis M, Nandi S et al (2006) ATP-binding cassette transporter A1 expression disrupts raft membrane microdomains through its ATPase-related functions. J Biol Chem 281:36091-36101

Lee MH, Hammad SM, Semler AJ et al (2010) HDL3, but not HDL2, stimulates plasminogen activator inhibitor-1 release from adipocytes: the role of sphingosine-1-phosphate. J Lipid Res 51:2619-2628

Levkau B (2013) Cardiovascular effects of sphingosine-1-phosphate (S1P). Handb Exp Pharmacol 216:147-170

Li Q, Yokoyama S (1995) Independent regulation of cholesterol incorporation into free apolipoprotein-mediated cellular lipid efflux in rat vascular smooth muscle cells. J Biol Chem 270:26216-26223

Li Q, Tsujita M, Yokoyama S (1997) Selective down-regulation by protein kinase C inhibitors of apolipoprotein-mediated cellular cholesterol efflux in macrophages. Biochemistry 36:1204512052

Liu D, Ji L, Tong X et al (2011) Human apolipoprotein A-I induces cyclooxygenase-2 expression and prostaglandin I-2 release in endothelial cells through ATP-binding cassette transporter A1. Am J Physiol Cell Physiol 301:C739-C748

Liu M, Seo J, Allegood J, Bi X et al (2013a) Hepatic ApoM overexpression stimulates formation of larger, ApoM/S1P-enriched plasma HDL. J Biol Chem 289:2801-2814

Liu XY, Lu Q, Ouyang XP et al (2013b) Apelin-13 increases expression of ATP-binding cassette transporter A1 via activating protein kinase $\mathrm{C} \alpha$ signaling in THP-1 macrophage-derived foam cells. Atherosclerosis 226:398-407

Ma L, Dong F, Denis M et al (2011) Ht31, a protein kinase A anchoring inhibitor, induces robust cholesterol efflux and reverses macrophage foam cell formation through ATP-binding cassette transporter A1. J Biol Chem 286:3370-3378

Maceyka M, Harikumar KB, Milstien S et al (2012) Sphingosine-1-phosphate signaling and its role in disease. Trends Cell Biol 22:50-60

Malaval C, Laffargue M, Barbaras R et al (2009) RhoA/ROCK I signalling downstream of the P2Y13 ADP-receptor controls HDL endocytosis in human hepatocytes. Cell Signal 21:120 127

Malchinkhuu E, Sato K, Muraki T et al (2003) Assessment of the role of sphingosine 1-phosphate and its receptors in high-density lipoprotein-induced stimulation of astroglial cell function. Biochem J 370:817-827

Martinez LO, Agerholm-Larsen B, Wang N et al (2003a) Phosphorylation of a pest sequence in ABCA1 promotes calpain degradation and is reversed by ApoA-I. J Biol Chem 278:3736837374

Martinez LO, Jacquet S, Esteve JP et al (2003b) Ectopic beta-chain of ATP synthase is an apolipoprotein A-I receptor in hepatic HDL endocytosis. Nature 421:75-79

Matsuo Y, Miura S, Kawamura A et al (2007) Newly developed reconstituted high-density lipoprotein containing sphingosine-1-phosphate induces endothelial tube formation. Atherosclerosis 194:159-168

McNeal CJ, Chatterjee S, Hou J et al (2013) Human HDL containing a novel apoC-I isoform induces smooth muscle cell apoptosis. Cardiovasc Res 98:83-93

Mendez AJ, Oram JF, Bierman EL (1991) Protein kinase C as a mediator of high density lipoprotein receptor-dependent efflux of intracellular cholesterol. J Biol Chem 266:1010410111

Mineo C, Shaul PW (2012) Functions of scavenger receptor class B, type I in atherosclerosis. Curr Opin Lipidol 23:487-493

Mineo C, Yuhanna IS, Quon MJ et al (2003) High density lipoprotein induced endothelial nitricoxide synthase activation is mediated by Akt and MAP kinases. J Biol Chem 278:9142-9149

Miura S, Fujino M, Matsuo Y et al (2003) High density lipoprotein-induced angiogenesis requires the activation of Ras/MAP kinase in human coronary artery endothelial cells. Arterioscler Thromb Vasc Biol 23:802-808 
Mogilenko DA, Orlov SV, Trulioff AS et al (2012) Endogenous apolipoprotein A-I stabilizes ATP-binding cassette transporter A1 and modulates Toll-like receptor 4 signaling in human macrophages. FASEB J 26:2019-2030

Morel S, Frias MA, Rosker C et al (2012) The natural cardioprotective particle HDL modulates connexin43 gap junction channels. Cardiovasc Res 93:41-49

Murphy AJ, Woollard KJ, Hoang A et al (2008) High-density lipoprotein reduces the human monocyte inflammatory response. Arterioscler Thromb Vasc Biol 28:2071-2077

Murphy AJ, Woollard KJ, Suhartoyo A et al (2011) Neutrophil activation is attenuated by highdensity lipoprotein and apolipoprotein A-I in in vitro and in vivo models of inflammation. Arterioscler Thromb Vasc Biol 31:1333-1341

Nofer JR, van Eck M (2011) HDL scavenger receptor class B type I and platelet function. Curr Opin Lipidol 22:277-282

Nofer J-R, Tepel M, Kehrel B et al (1996) High density lipoproteins enhance the $\mathrm{Na}^{+} / \mathrm{H}^{+}$antiport in human platelets. Thromb Haemost 75:635-641

Nofer JR, Walter M, Kehrel B et al (1998) HDL3-mediated inhibition of thrombin-induced platelet aggregation and fibrinogen binding occurs via decreased production of phosphoinositidederived second messengers 1,2-diacylglycerol and inositol 1,4,5-tris-phosphate. Arterioscler Thromb Vasc Biol 18:861-869

Nofer JR, Fobker M, Höbbel G et al (2000) Activation of phosphatidylinositol-specific phospholipase $\mathrm{C}$ by HDL-associated lysosphingolipid. Involvement in mitogenesis but not in cholesterol efflux. Biochemistry 39:15199-15207

Nofer JR, Junker R, Pulawski E et al (2001a) High density lipoproteins induce cell cycle entry in vascular smooth muscle cells via mitogen activated protein kinase-dependent pathway. Thromb Haemost 85:730-735

Nofer JR, Levkau B, Wolinska I et al (2001b) Suppression of endothelial cell apoptosis by high density lipoproteins (HDL) and HDL-associated lysosphingolipids. J Biol Chem 276:34480 34485

Nofer JR, Feuerborn R, Levkau B et al (2003) Involvement of Cdc42 signaling in apoA-I-induced cholesterol efflux. J Biol Chem 278:53055-53062

Nofer JR, van der Giet M, Tölle M et al (2004) HDL induces NO-dependent vasorelaxation via the lysophospholipid receptor S1P3. J Clin Invest 113:569-581

Nofer JR, Remaley AT, Feuerborn R et al (2006) Apolipoprotein A-I activates Cdc42 signaling through the ABCA1 transporter. J Lipid Res 47:794-803

Nofer JR, Brodde M, Korporaal SJ et al (2011) Native high density lipoproteins inhibit thrombininduced platelet activation via scavenger receptor B1 (SR-B1). Atherosclerosis 215:374-382

Norata GD, Callegari E, Marchesi M et al (2005) High-density lipoproteins induce transforming growth factor-beta2 expression in endothelial cells. Circulation 111:2805-2811

Norata GD, Marchesi P, Pirillo A et al (2008) Long pentraxin 3, a key component of innate immunity, is modulated by high density lipoproteins in endothelial cells. Arterioscler Thromb Vasc Biol 28:925-931

Paatela H, Vihma V, Jauhiainen M et al (2011) Dehydroepiandrosterone fatty acyl esters in high density lipoprotein: interaction with human vascular endothelial cells and vascular responses ex vivo. Steroids 76:376-380

Radojkovic C, Genoux A, Pons V et al (2009) Stimulation of cell surface F1-ATPase activity by apolipoprotein A-I inhibits endothelial cell apoptosis and promotes proliferation. Arterioscler Thromb Vasc Biol 29:1125-1130

Riwanto M, Rohrer L, Roschitzki B et al (2013) Altered activation of endothelial anti- and proapoptotic pathways by high-density lipoprotein from patients with coronary artery disease: role of high-density lipoprotein-proteome remodeling. Circulation 127:891-904

Rust S, Rosier M, Funke H et al (1999) Tangier disease is caused by mutations in the gene encoding ATP-binding cassette transporter 1. Nat Genet 22:352-355

Sachinidis A, Kettenhofen R, Seewald S et al (1999) Evidence that lipoproteins are carriers of bioactive factors. Arterioscler Thromb Vasc Biol 19:2412-2421 
Saddar S, Carriere V, Lee WR et al (2013) Scavenger receptor class B type I is a plasma membrane cholesterol sensor. Circ Res 112:140-151

Sano O, Kobayashi A, Nagao K et al (2007) Sphingomyelin-dependence of cholesterol efflux mediated by ABCG1. J Lipid Res 48:2377-2384

Sato K, Malchinkhuu E, Horiuchi Y et al (2007) HDL-like lipoproteins in cerebrospinal fluid affect neural cell activity through lipoprotein-associated sphingosine 1-phosphate. Biochem Biophys Res Commun 359:649-654

Sattler KJ, Elbasan S, Keul P et al (2010) Sphingosine 1-phosphate levels in plasma and HDL are altered in coronary artery disease. Basic Res Cardiol 105:821-832

See RH, Caday-Malcolm RA, Singaraja RR et al (2002) Protein kinase A site-specific phosphorylation regulates ATP-binding cassette A1 (ABCA1)-mediated phospholipid efflux. J Biol Chem 277:41835-41842

Seetharam D, Mineo C, Gormley AK et al (2006) High-density lipoprotein promotes endothelial cell migration and reendothelialization via scavenger receptor-B type I. Circ Res 98:63-72

Sekine Y, Suzuki K, Remaley AT (2011) HDL and sphingosine-1-phosphate activate stat3 in prostate cancer DU145 cells via ERK1/2 and S1P receptors, and promote cell migration and invasion. Prostate 71:690-699

Slotte JP, Oram JF, Bierman EL (1987) Binding of high density lipoproteins to cell receptors promotes translocation of cholesterol from intracellular membranes to the cell surface. J Biol Chem 262:12904-12907

Speer T, Rohrer L, Blyszczuk P et al (2013) Abnormal high-density lipoprotein induces endothelial dysfunction via activation of toll-like receptor-2. Immunity 38:754-768

Sun Y, Ishibashi M, Seimon T et al (2009) Free cholesterol accumulation in macrophage membranes activates Toll-like receptors and p38 mitogen-activated protein kinase and induces cathepsin K. Circ Res 104:455-465

Tamama K, Tomura H, Sato K et al (2005) High density lipoprotein inhibits migration of vascular smooth muscle cells through its sphingosine 1-phosphate component. Atherosclerosis 178:1923

Tan JT, Prosser HC, Vanags LZ et al (2013) High-density lipoproteins augment hypoxia-induced angiogenesis via regulation of post-translational modulation of hypoxia-inducible factor $1 \alpha$. FASEB J 28:206-217

Tang C, Vaughan AM, Oram JF (2004) Janus kinase 2 modulates the apolipoprotein interactions with ABCA1 required for removing cellular cholesterol. J Biol Chem 279:7622-7628

Tang C, Vaughan AM, Anantharamaiah GM et al (2006) Janus kinase 2 modulates the lipidremoving but not protein-stabilizing interactions of amphipathic helices with ABCA1. J Lipid Res 47:107-114

Tang C, Liu Y, Kessler PS, Vaughan AM, Oram JF (2009) The macrophage cholesterol exporter ABCA1 functions as an anti-inflammatory receptor. J Biol Chem 284:32336-32343

Tao R, Hoover HE, Honbo N et al (2010) High-density lipoprotein determines adult mouse cardiomyocyte fate after hypoxia-reoxygenation through lipoprotein-associated sphingosine 1-phosphate. Am J Physiol Heart Circ Physiol 298:H1022-H1028

Tatematsu S, Francis SA, Natarajan P et al (2013) Endothelial lipase is a critical determinant of high-density lipoprotein-stimulated sphingosine 1-phosphate-dependent signaling in vascular endothelium. Arterioscler Thromb Vasc Biol 33:1788-1794

Tölle M, Pawlak A, Schuchardt M et al (2008) HDL-associated lysosphingolipids inhibit NAD(P) $\mathrm{H}$ oxidase-dependent monocyte chemoattractant protein-1 production. Arterioscler Thromb Vasc Biol 28:1542-1548

Tölle M, Huang T, Schuchardt M et al (2012) High-density lipoprotein loses its anti-inflammatory capacity by accumulation of pro-inflammatory-serum amyloid A. Cardiovasc Res 94:154-162

Tsukamoto K, Hirano K, Tsujii K et al (2001) ATP-binding cassette transporter-1 induces rearrangement of actin cytoskeletons possibly through Cdc42/N-WASP. Biochem Biophys Res Commun 287:757-765 
Tsukamoto K, Hirano K, Yamashita S et al (2002) Retarded intracellular lipid transport associated with reduced expression of Cdc42, a member of Rho-GTPases, in human aged skin fibroblasts: a possible function of $\mathrm{Cdc} 42$ in mediating intracellular lipid transport. Arterioscler Thromb Vasc Biol 22:1899-1904

Umemoto T, Han CY, Mitra P et al (2013) Apolipoprotein AI and high-density lipoprotein have anti-inflammatory effects on adipocytes via cholesterol transporters: ATP-binding cassette A-1, ATP-binding cassette G-1, and scavenger receptor B-1. Circ Res 112:1345-1354

Vaughan AM, Tang C, Oram JF (2009) ABCA1 mutants reveal an interdependency between lipid export function, apoA-I binding activity, and Janus kinase 2 activation. J Lipid Res 50:285-292

von Otte S, Paletta JR, Becker S et al (2006) Follicular fluid high density lipoprotein-associated sphingosine 1-phosphate is a novel mediator of ovarian angiogenesis. J Biol Chem 281:53985405

Walter M, Reinecke H, Nofer JR et al (1995) HDL3 stimulates multiple signaling pathways in human skin fibroblasts. Arterioscler Thromb Vasc Biol 15:1975-1986

Walter M, Reinecke H, Gerdes U et al (1996) Defective regulation of phosphatidylcholine-specific phospholipases $\mathrm{C}$ and $\mathrm{D}$ in a kindred with Tangier disease. Evidence for the involvement of phosphatidylcholine breakdown in HDL-mediated cholesterol efflux mechanisms. J Clin Invest 98:2315-2323

Wang N, Silver DL, Costet P, Tall AR (2000) Specific binding of ApoA-I, enhanced cholesterol efflux, and altered plasma membrane morphology in cells expressing $\mathrm{ABC} 1$. J Biol Chem 275:33053-33058

Wang N, Chen W, Linsel-Nitschke P et al (2003) A PEST sequence in ABCA1 regulates degradation by calpain protease and stabilization of ABCA1 by apoA-I. J Clin Invest 111:99-107

Wang N, Lan D, Chen W et al (2004) ATP-binding cassette transporters G1 and G4 mediate cellular cholesterol efflux to high-density lipoproteins. Proc Natl Acad Sci USA 101:97749779

Wang N, Ranalletta M, Matsuura F et al (2006) LXR-induced redistribution of ABCG1 to plasma membrane in macrophages enhances cholesterol mass efflux to HDL. Arterioscler Thromb Vasc Biol 26:1310-1316

Weichhart T, Kopecky C, Kubicek M et al (2012) Serum amyloid A in uremic HDL promotes inflammation. J Am Soc Nephrol 23:934-947

Westerterp M, Gourion-Arsiquaud S, Murphy AJ et al (2012) Regulation of hematopoietic stem and progenitor cell mobilization by cholesterol efflux pathways. Cell Stem Cell 11:195-206

Wilhelm AJ, Zabalawi M, Grayson JM et al (2009) Apolipoprotein A-I and its role in lymphocyte cholesterol homeostasis and autoimmunity. Arterioscler Thromb Vasc Biol 29:843-849

Wilkerson BA, Grass GD, Wing SB et al (2012) Sphingosine 1-phosphate (S1P) carrier-dependent regulation of endothelial barrier: high density lipoprotein (HDL)-S1P prolongs endothelial barrier enhancement as compared with albumin-S1P via effects on levels, trafficking, and signaling of S1P1. J Biol Chem 287:44645-44653

Xu J, Qian J, Xie X et al (2012) High density lipoprotein cholesterol promotes the proliferation of bone-derived mesenchymal stem cells via binding scavenger receptor-B type I and activation of PI3K/Akt, MAPK/ERK1/2 pathways. Mol Cell Biochem 371:55-64

Yamauchi Y, Hayashi M, Abe-Dohmae S et al (2003) Apolipoprotein A-I activates protein kinase $\mathrm{C}$ alpha signaling to phosphorylate and stabilize ATP binding cassette transporter A1 for the high density lipoprotein assembly. J Biol Chem 278:47890-47897

Yamauchi Y, Chang CC, Hayashi M et al (2004) Intracellular cholesterol mobilization involved in the ABCA1/apolipoprotein-mediated assembly of high density lipoprotein in fibroblasts. J Lipid Res 45:1943-1951

Yin K, Deng X, Mo ZC et al (2011) Tristetraprolin-dependent post-transcriptional regulation of inflammatory cytokine mRNA expression by apolipoprotein A-I: role of ATP-binding membrane cassette transporter A1 and signal transducer and activator of transcription 3. J Biol Chem 286:13834-13845 
Yin K, Chen WJ, Zhou ZG et al (2012) Apolipoprotein A-I inhibits CD40 proinflammatory signaling via ATP-binding cassette transporter A1-mediated modulation of lipid raft in macrophages. J Atheroscler Thromb 19:823-836

Yuhanna IS, Zhu Y, Cox BE et al (2001) High-density lipoprotein binding to scavenger receptorBI activates endothelial nitric oxide synthase. Nat Med 7:853-857

Yvan-Charvet L, Welch C, Pagler TA et al (2008) Increased inflammatory gene expression in $\mathrm{ABC}$ transporter-deficient macrophages: free cholesterol accumulation, increased signaling via toll-like receptors, and neutrophil infiltration of atherosclerotic lesions. Circulation 118:18371847

Yvan-Charvet L, Pagler TA, Seimon TA et al (2010a) ABCA1 and ABCG1 protect against oxidative stress-induced macrophage apoptosis during efferocytosis. Circ Res 106:1861-1869

Yvan-Charvet L, Pagler T, Gautier EL et al (2010b) ATP-binding cassette transporters and HDL suppress hematopoietic stem cell proliferation. Science 328:1689-1693

Zhang Z, Hirano K, Tsukamoto K et al (2005) Defective cholesterol efflux in Werner syndrome fibroblasts and its phenotypic correction by Cdc42, a RhoGTPase. Exp Gerontol 40:286-294

Zhang Q, Zhang Y, Feng H et al (2011) High density lipoprotein (HDL) promotes glucose uptake in adipocytes and glycogen synthesis in muscle cells. PLoS ONE 6:e23556

Zhang QH, Zu XY, Cao RX et al (2012) An involvement of SR-B1 mediated PI3K-Akt-eNOS signaling in HDL-induced cyclooxygenase 2 expression and prostacyclin production in endothelial cells. Biochem Biophys Res Commun 420:17-23

Zhu X, Lee JY, Timmins JM et al (2008a) Increased cellular free cholesterol in macrophagespecific Abca1 knock-out mice enhances pro-inflammatory response of macrophages. J Biol Chem 283:22930-22941

Zhu W, Saddar S, Seetharam D et al (2008b) The scavenger receptor class B type I adaptor protein PDZK1 maintains endothelial monolayer integrity. Circ Res 102:480-487 